\title{
Erratum to: Total Fluid Intake and the Risk of Recurrence in Patients with Non-Muscle Invasive Bladder Cancer: A Prospective Cohort Study
}

\author{
Sylvia H.J. Jochems ${ }^{\mathrm{a}, \mathrm{b}, *}$, Anke Wesselius ${ }^{\mathrm{b}}$ and Maurice P. Zeegers ${ }^{\mathrm{a}, \mathrm{b}}$ \\ ${ }^{a}$ Institute of Cancer and Genomic Sciences, University of Birmingham, Birmingham, UK \\ ${ }^{\mathrm{b}}$ Department of Complex Genetics and Epidemiology, School of Nutrition and Translational Research \\ in Metabolism, Maastricht University, Maastricht, The Netherlands
}

Received 24 May 2020

Accepted 26 May 2020

Pre-press 19 June 2020

Published 21 September 2020

The FFQ used in this study may have led to underestimation of the absolute daily fluid intake from beverages. Therefore, the absolute values for total fluid intake and total alcohol intake should not be interpreted as such. Nonetheless, no differential misclassification of intake was observed between patients with and without a recurrence of bladder cancer and sensitivity analyses indicated a correct ranking of the patients in this study.

In addition, in the footnote of Tables S1 and S2 in the supplemental material of our article (1) the sentence 'Q1 includes non-drinkers only, Q2 includes drinkers whose consumption corresponds with up to 1 large glass of wine or half a pint of beer/lager per day, Q3 includes drinkers whose consumption corresponds with more than 1 large glass of wine or half a pint of beer/cider per day' should be adjusted to: 'T1 includes non-drinkers only, $\mathrm{T} 2$ includes drinkers with the lowest consumption of all included alcoholic beverages, $\mathrm{T} 3$ includes drinkers with the highest consumption of all included alcoholic beverages'.

\section{REFERENCES}

[1] Jochems SHJ, van Osch FHM, Reulen RC, van Hensbergen M, Nekeman D, Pirrie S, Wesselius A, van Schooten FJ, James ND, Wallace DMA, Bryan RT, Cheng KK, Zeegers MP. Total fluid intake and the risk of recurrence in patients with non-muscle invasive bladder cancer: a prospective cohort study - Bladder Cancer. 2018;4(3):303-10. doi: 10.3233/BLC-180172.

${ }^{*}$ Correspondence to: Sylvia H.J. Jochems, Maastricht University, Department of Complex Genetics and Epidemiology, PO Box 616, 6200 MD Maastricht, The Netherlands. E-mail: s.jochems@maastrichtuniversity.nl. 